\title{
Skyrmion Dynamics at Finite Temperatures: Beyond Thiele's Equation
}

\author{
Markus Weißenhofer®, ${ }^{*}$ Levente Rózsa๑, and Ulrich Nowak® \\ Fachbereich Physik, Universität Konstanz, DE-78457 Konstanz, Germany
}

(Received 12 April 2021; revised 10 June 2021; accepted 21 June 2021; published 22 July 2021)

\begin{abstract}
Magnetic textures are often treated as quasiparticles following Thiele's equation of motion. We demonstrate via spin model simulations of the current-driven and Brownian motion of ferromagnetic skyrmions that the existing theory based on Thiele's equation is insufficient to describe the dynamics of skyrmions at finite temperatures. We propose an extended equation of motion that goes beyond Thiele's equation by taking into account the coupling of the skyrmion to the magnonic heat bath leading to an additional dissipative term that is linear in temperature. Our results indicate that this so-far-neglected magnon-induced friction dominates for finite temperatures and Gilbert damping values typical for thin films and multilayers.
\end{abstract}

DOI: 10.1103/PhysRevLett.127.047203

Isolated magnetic skyrmions are topologically nontrivial spin configurations that are embedded in homogeneous magnetic phases. The directions of the magnetic moments $S$ span the whole unit sphere, giving rise to a finite topological charge $Q=1 /(4 \pi) \int d^{2} r S \cdot\left(\partial_{x} S \times\right.$ $\left.\partial_{y} S\right)$ [1-3]. As they can easily be manipulated by magnetic, optical, and electrical means, skyrmions are promising candidates as elements in conventional [4] and unconventional computing [5,6] architectures. Since the first detection of isolated skyrmions [7], they have attracted considerable research interest, resulting in experimental measurements of their current-driven dynamics [8-11] and temperature-induced Brownian motion [12-14].

So far, isolated skyrmions have often been treated as quasiparticles and, in terms of a rigid-body description, their dynamics has been analyzed via the so-called Thiele equation [15]. This approach has proven successful in describing the current-driven motion [16,17] of skyrmions, yielding quantitative agreement with numerical simulations at zero temperature. Furthermore, it leads to the prediction of skyrmion Brownian motion and the derivation of an analytic expression for the diffusion coefficients [18-20] whose validity in the high damping and/or low temperature regime was demonstrated using spin model simulations [21,22]. In experiments, however, the situation is frequently the opposite: the damping can be rather low and temperatures are usually rather high. These limits are of special importance for potential applications of skyrmions because it is desirable that potential devices operate at room temperatures and in low-pinning materials. The interplay between currentdriven motion and elevated temperatures, although highly relevant for applications, has so far only been investigated experimentally [11] and a theoretical discussion is still lacking.
It was shown in the general context of solitons that at finite temperatures the low-lying excitations that are thermally activated serve as a heat bath to the soliton [23]. In the case of spin systems, the low-lying excitations are the magnons. Numerical calculations [24] and analytical investigations within a continuum model [25] demonstrated that skyrmions create a finite scattering potential for the magnons and that momentum is transferred from the magnons to the skyrmion. Hence, at finite temperatures the magnons serve as a heat bath that is coupled with the skyrmion dynamics. The impact of this additional heat bath has been studied for the quantum dynamics of domain walls [26,27], vortices [28], and skyrmions [29,30] and was found to give rise to a magnon-induced friction.

This Letter is organized as follows: first, we demonstrate by means of an atomistic spin model approach and the stochastic Landau-Lifshitz-Gilbert equation that the existing theory based on Thiele's equation is insufficient to describe the Brownian motion of skyrmionic structures with various topological charges at elevated temperatures. We then propose an effective finite-temperature equation of motion that goes beyond the Thiele equation by taking into account the coupling of the skyrmions to the magnonic heat bath via an additional friction term that is linear in temperature. Comparing our model to spin model simulations, we obtain an estimate for the strength of this additional magnon-induced friction for skyrmions with various topological charges. Last, we explore the impact of the magnon-induced friction on the current-driven motion via the spin-orbit torque.

The derivation of the Thiele equation rests on a spatial average of microscopic spin degrees of freedom in a rigid body approximation [15]. To test its validity, we chose a spin model for a $\left(\mathrm{Pt}_{0.95} \mathrm{Ir}_{0.05}\right) / \mathrm{Fe}$ bilayer on a $\operatorname{Pd}(111)$ surface via a spin Hamiltonian $\mathcal{H}$ considering only the magnetic Fe moments, 


$$
\mathcal{H}=\frac{1}{2} \sum_{i \neq j} \boldsymbol{S}_{i} \mathcal{J}_{i j} \boldsymbol{S}_{j}+\sum_{i} \boldsymbol{S}_{i} \mathcal{K} \boldsymbol{S}_{i}-\sum_{i} \mu_{s} \boldsymbol{S}_{i} \cdot \boldsymbol{B}
$$

The magnetic spin moment $\mu_{s}=3.06 \times 10^{-23} \mathrm{~J} / \mathrm{T}$, the onsite anisotropy tensor $\mathcal{K}$, and the tensorial exchange coefficients $\mathcal{J}_{i j}$, which model the Heisenberg exchange, Dzyaloshinsky-Moriya interaction (DMI), and the two-site anisotropy, were taken from Refs. [12,31]. $\boldsymbol{B}$ is an external magnetic field that is applied perpendicularly to the surface and has a fixed value of $B_{\perp}=0.5 \mathrm{~T}$ throughout the study. At $B_{\perp}=0 \mathrm{~T}$, the ground state of the system is a spin spiral state that transforms into a collinear state if a field of $B_{\perp} \geq$ $0.21 \mathrm{~T}$ is applied [32]. Skyrmionic spin structures with different topological charges $Q$ can then occur as metastable excitations. So far, skyrmionic spin structures with topological charges ranging from -3 to 3 have been identified in this system [33]. The DMI, the antisymmetric part of the $\mathcal{J}_{i j}$, in this system prefers the formation of Néel-type skyrmionic spin structures with $Q=1$. The spin structures with other topological charges are stabilized by the frustration of the Heisenberg exchange, despite being deformed by the DMI [32]. Unless stated otherwise, all the results presented in the following were obtained for the usual $Q=1$ skyrmionic spin structure, which will be simply referred to as skyrmion in what follows. The dynamics of the spins is calculated by the means of the stochastic Landau-Lifshitz-Gilbert (LLG) equation of motion [34-36],

$\frac{\partial \boldsymbol{S}_{i}}{\partial t}=-\frac{\gamma}{\left(1+\alpha^{2}\right) \mu_{s}} \boldsymbol{S}_{i} \times\left(\left[\boldsymbol{H}_{i}+\boldsymbol{\zeta}_{i}\right]+\alpha \boldsymbol{S}_{i} \times\left[\boldsymbol{H}_{i}+\boldsymbol{\zeta}_{i}\right]\right)+\boldsymbol{T}_{i}^{\mathrm{SOT}}$,

where $\gamma=1.76 \times 10^{11} \mathrm{~s}^{-1} \mathrm{~T}^{-1}$ is the absolute value of the gyromagnetic ratio and $\alpha$ is the Gilbert damping. $\boldsymbol{H}_{i}=$ $-\partial \mathcal{H} / \partial \boldsymbol{S}_{i}$ is the effective field that is generated by the external field, the on-site anisotropy, and the interactions with the neighboring spins. $\boldsymbol{\zeta}_{i}$ denotes a stochastic field modeling the effects of thermal fluctuations. Following Ref. [37], $\zeta_{i}$ has zero mean, and its autocorrelation is given by $\left\langle\zeta_{i}^{\mu}(t) \zeta_{j}^{\nu}\left(t^{\prime}\right)\right\rangle=2 \alpha \mu_{\mathrm{s}} k_{B} T \delta_{\mu \nu} \delta_{i j} \delta\left(t-t^{\prime}\right) / \gamma$, where $\mu$ and $\nu$ are Cartesian components and $k_{B}$ is the Boltzmann constant. In order to include the effect of spin-polarized currents on spin textures, the right-hand side of Eq. (2) is supplemented with the spin-orbit torque term [38,39]

$$
\boldsymbol{T}_{i}^{\text {SOT }}=\frac{\beta_{f}-\alpha \beta_{d}}{1+\alpha^{2}} \boldsymbol{S}_{i} \times \boldsymbol{P}+\frac{\alpha \beta_{f}+\beta_{d}}{1+\alpha^{2}} \boldsymbol{S}_{i} \times\left(\boldsymbol{S}_{i} \times \boldsymbol{P}\right),
$$

where $\boldsymbol{P}$ is the effective spin polarization direction and $\beta_{f}$ and $\beta_{d}$ are the strength of the fieldlike and dampinglike torque, respectively, that depend on the injected current density.
The numerical integration of the stochastic LLG equation is performed via a GPU-based implementation of Heun's algorithm [36]. We simulate $512 \times 512$ spins featuring periodic boundary conditions with a fixed timestep $\Delta t=1.4 \times 10^{-15} \mathrm{~s}$ for $\alpha \geq 0.005, \Delta t=7 \times 10^{-16} \mathrm{~s}$ for $0.001 \leq \alpha<0.005$, and $\Delta t=1.4 \times 10^{-16} \mathrm{~s}$ for $\alpha<0.001$. The trajectories $\boldsymbol{r}(t)$ of skyrmionic spin structures were determined by monitoring the change in the outof-plane magnetization, which has proven robust against thermal fluctuations [18].

It is usually assumed that the motion of skyrmionic spin structures can be described in terms of a rigid-body description via the so-called Thiele equation [15] that can be derived from the LLG equation reading

$$
\boldsymbol{G} \times \boldsymbol{v}+\Gamma \boldsymbol{v}=\boldsymbol{F} .
$$

Here, $\boldsymbol{F}$ represents forces exerted on the skyrmionic spin structures, $\boldsymbol{v}$ is its velocity, $\boldsymbol{G}=-4 \pi Q \mu_{s} / \gamma a^{2} \boldsymbol{e}_{\perp}$ is the gyrocoupling vector, $\Gamma$ describes friction, and $a=2.751 \times$ $10^{-10} \mathrm{~m}$ is the lattice constant. Within the framework of the Thiele equation, $\Gamma=\alpha \mathfrak{D}$ with $\mathfrak{D}=\mu_{s} /\left(2 \gamma a^{2}\right) \int d^{2} r\left(\partial_{x} \boldsymbol{S}\right.$. $\left.\partial_{x} \boldsymbol{S}+\partial_{y} \boldsymbol{S} \cdot \partial_{y} \boldsymbol{S}\right)$ and quantifying friction of the skyrmionic motion by dissipation of energy to the electronic and/or phononic subsystems. This type of friction is proportional to the Gilbert damping, and thus Eq. (4) predicts undamped motion in the limit of vanishing $\alpha$. The first term in Eq. (4) is a result of the nontrivial topology of the skyrmionic spin structures and gives rise to motion that is transverse to the direction of the force.

The specifics of the effective force $\boldsymbol{F}$ depend on the driving mechanism. It was demonstrated in Ref. [21] that finite-temperature fluctuations due to the coupling to the electronic and/or phononic heat bath, which are modeled via the stochastic field $\zeta_{i}$ in Eq. (2), lead to a stochastic effective force $\boldsymbol{F}^{\text {th }}$. This force has zero mean, and its autocorrelation is given by $\left\langle F_{\mu}^{\text {th }}(t) F_{\nu}^{\text {th }}\left(t^{\prime}\right)\right\rangle=$ $2 k_{B} T \Gamma \delta_{\mu \nu} \delta\left(t-t^{\prime}\right)$. Equation (4) then becomes a Langevin-type equation of motion. The diffusion coefficient $D$ can be calculated from the mean-squared displacement via $\left\langle[\boldsymbol{r}(t)-\boldsymbol{r}(0)]^{2}\right\rangle=2 D t$ and reads $[18,19,22]$

$$
D=k_{B} T \frac{\Gamma}{\Gamma^{2}+G^{2}} .
$$

This expression indicates a peculiar dependence on friction. Usually, the diffusion coefficient of a particle increases if its friction is lowered since typically $D=k_{B} T / \Gamma$. For skyrmionic spin structures, the situation is opposite: due to the gyrocoupling term in Eq. (4), diffusive motion decreases with decreasing friction [18]. So far, Eq. (5) has been successful in predicting the diffusion coefficients of skyrmions by yielding quantitative agreement with numerical simulations $[21,22]$. However, these previous 


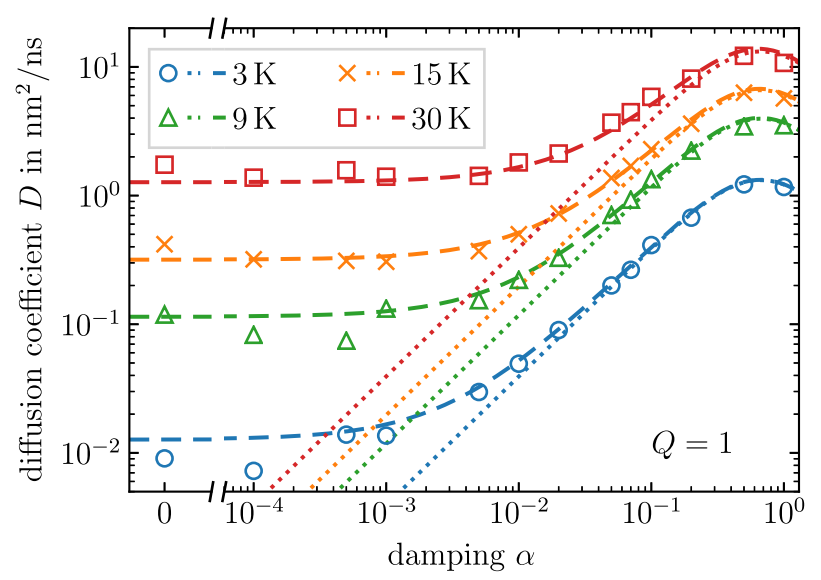

FIG. 1. Diffusion coefficient $D$ versus $\alpha$ for various temperatures. Symbols are simulation results that are compared to the theoretical predictions based on the normal Thiele equation [Eq. (5)] (dotted lines) and our extended model [Eq. (7)] with $\eta=5.05 \times 10^{-17} \mathrm{~kg} /(\mathrm{sK})$ (dashed lines).

investigations were restricted to comparably large values for $\alpha$ and/or low temperatures.

In the following, we investigate the Brownian motion of skyrmions for a wide range of values for $\alpha$ (including the limit $\alpha \rightarrow 0$ ) and for high temperatures. Note that, especially for $\alpha=0$, the spin system is decoupled from the heat bath and its energy is conserved. The dynamics are then described within the microcanonical ensemble, where the definition of temperature is subtle. The following results for $\alpha=0$ were obtained by first equilibrating the system with finite $\alpha$ at different temperatures and then decoupling the system from the heat bath.

Figure 1 depicts the diffusion coefficients obtained by calculating the mean-squared displacements from simulation data for different values of $T \leq 30 \mathrm{~K}$ (the critical temperature of the system is approximately $50 \mathrm{~K}$ [40]) and for $\alpha \in[0,1]$. The dotted lines correspond to Eq. (5) and compare favorably only for $\alpha \gtrsim 0.1$. For example, for $\alpha=10^{-3}$, the estimate for the diffusion coefficient is more than 1 order of magnitude lower than the values obtained from the simulation data. The most striking difference between the numerical results and Eq. (5) can be observed in the limit $\alpha \rightarrow 0$ : the diffusion coefficients do not vanish; instead, we find that the numerically obtained $D$ converge to constant values that only depend on temperature.

The fact that Eq. (5) fails to describe the behavior in the low $\alpha$ limit indicates that the Thiele equation [Eq. (4)] has to be extended by an additional term in order to be valid at finite temperatures. We propose that this term originates from the presence of magnons in the spin system at finite temperatures that serve as an additional heat bath [26-30]. A heuristic discussion of the interplay between magnons and skyrmion dynamics will be given in what follows (for a sketch illustrating our line of reasoning, see Fig. 2). Finite temperatures lead to the occupation of low-lying collective

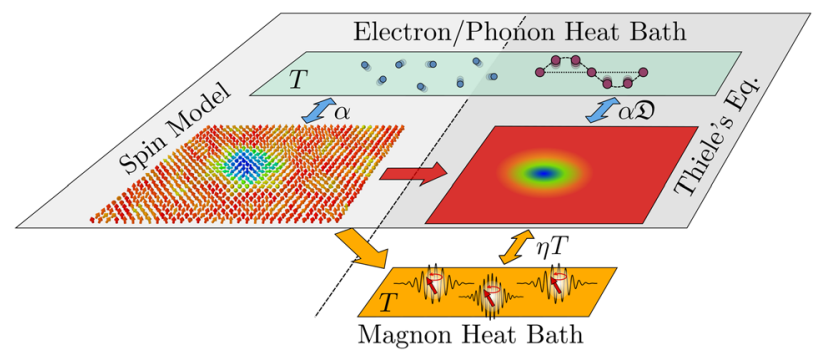

FIG. 2. Sketch of the transformation of a spin model to an effective rigid-body description: in the spin model (left part), the system is connected to the electronic or phononic heat bath via the Gilbert damping $\alpha$, which results in the $\alpha \mathfrak{D}$ term in the Thiele equation (right part). At finite temperatures, magnons are present in the spin model whose impact has to be accounted for by an additional $\eta T$ term in the effective rigid-body description.

excitations of the spin system, the magnons, which propagate through the system. As the skyrmion creates a finite scattering potential $[24,25]$, there is a coupling of the skyrmion motion to the magnons in the system. This leads to a damping of the skyrmion by dissipation of energy to the magnon heat bath. The simplest way to extend the Thiele equation is by replacing the friction in Eq. (4) with an effective friction $\Gamma^{\text {eff }}$ via

$$
\boldsymbol{G} \times \boldsymbol{v}+\underbrace{(\alpha \mathfrak{D}+\eta T)}_{\text {reff }} \boldsymbol{v}=\boldsymbol{F} .
$$

Here, we assume that, for symmetry reasons, the magnoninduced friction $\eta T$ is isotropic and consequently $\eta$ is a scalar quantity. Linearity in $T$ is concluded from the fact that in the classical description the magnon occupation is proportional to temperature [41]. Note, however, that the scaling with temperature depends on whether one treats the magnons as classical or quantum excitation [29]. Moreover, since the occupation of the magnon modes is an equilibrium property, we further assume that $\eta$ is independent of $\alpha$. This has profound consequences: depending on the respective values of $\mathfrak{D}$ and $\eta$, there is a range of $\alpha$ and $T$ for which the damping of the skyrmion motion due to coupling to the electronic or phononic system becomes negligible and the dynamics are fully governed by the coupling to the magnon heat bath. This is especially important in the limit of vanishing $\alpha$, where the damping of the motion of skyrmions remains finite for $T>0$.

Let us now reconsider the Brownian motion of skyrmions. Assuming that the fluctuation-dissipation theorem [42] is fulfilled, Eq. (6) is supplemented with an additional stochastic force whose autocorrelation depends on $\eta T$, and the diffusion coefficient follows, analogous to Eq. (5), as

$$
D=k_{B} T \frac{\Gamma^{\mathrm{eff}}}{\left(\Gamma^{\mathrm{eff}}\right)^{2}+G^{2}} .
$$

The above formula reduces to Eq. (5) for $\eta=0$. For nonzero $\eta$, Eq. (7) describes the Brownian motion of 


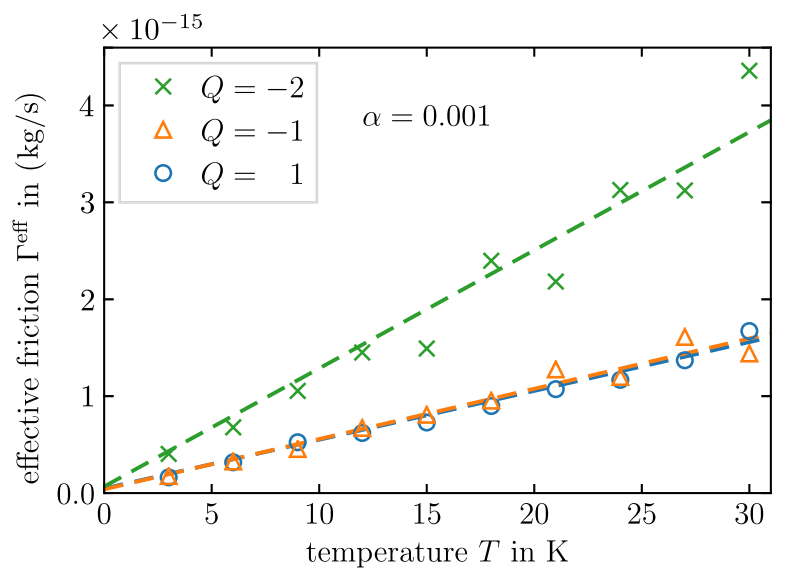

FIG. 3. Numerically obtained effective friction $\Gamma^{\text {eff }}$ versus $T$ for different types of skyrmionic spin structures. Dashed lines correspond to linear fits yielding $\eta \approx 5.05 \times 10^{-17} \mathrm{~kg} /(\mathrm{sK})$ for skyrmionic spin structures with $Q= \pm 1$ and $\eta \approx 1.22 \times$ $10^{-16} \mathrm{~kg} /(\mathrm{sK})$ for $Q=-2$ structures. The offset at $T=0$ corresponds to $\alpha \mathfrak{D}$.

skyrmions that interact with two types of reservoirs, the phononic or electronic contributions (which depend on $\alpha$ and are linear in $T$ ) and the magnonic contributions (independent of $\alpha$ and proportional to $T^{2}$ ).

We will now demonstrate the validity of our ansatz for the magnon-induced friction in Eq. (6) and calculate numerically the phenomenologically introduced parameter $\eta$. Using Eq. (7), we can calculate the effective friction via $\Gamma^{\mathrm{eff}}=k_{B} T /(2 D)-\sqrt{\left[k_{B} T /(2 D)\right]^{2}-G^{2}}$. Figure 3 shows the effective friction of skyrmionic spin structures with $Q=-2,-1,1$ (for a figure depicting their spin structures, see Ref. [43]) obtained this way from the numerical results for the diffusion coefficient. In agreement with our proposed generalization of the Thiele equation, we find a small offset at $T=0$ corresponding to $\alpha \mathfrak{D}$ and a linear dependence on temperature. As the offset is insignificant, Fig. 3 again demonstrates that at low $\alpha$ and finite temperatures the magnon-induced friction dominates and the damping resulting from a coupling to the electronic or phononic heat bath is negligible. We further find that the slope is $Q$ dependent: while the magnon-induced friction is quite similar for skyrmionic spin structures with $Q= \pm 1$, it is approximately 2 times larger for the $Q=-2$ structure. Using a linear fit, we can estimate the strength of the magnon-induced friction and obtain $\eta(Q= \pm 1) \approx 5.05 \times$ $10^{-17} \mathrm{~kg} /(\mathrm{sK})$ and $\eta(Q=-2) \approx 1.22 \times 10^{-16} \mathrm{~kg} /(\mathrm{sK})$. Revisiting Fig. 1, we can now compare the predictions based on our extended Thiele equation (dashed lines) [see Eq. (7)] with the numerically obtained values for the diffusion coefficients. They compare favorably, indicating that $\eta$ is indeed independent of $\alpha$ and $T$.

Since we have shown that finite temperatures require extensions to the Thiele equation in order to capture the diffusive dynamics of skyrmions, the question arises as to

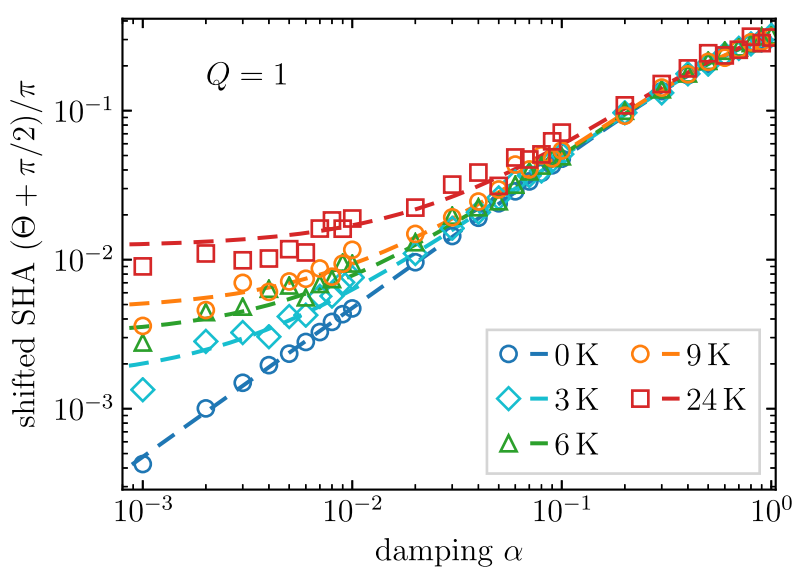

FIG. 4. Skyrmion Hall angle $\Theta$, shifted by $\pi / 2$, versus $\alpha$ for various values of $T$. Symbols are from simulations that are compared to the theoretical predictions based on Eq. (8) with $\eta=5.05 \times 10^{-17} \mathrm{~kg} /(\mathrm{sK})$ (dashed lines).

whether similar effects can be observed for skyrmions driven by other means, e.g., electrical currents. Hence, we finally investigate the finite-temperature current-driven motion of skyrmions via spin-orbit torques [see Eq. (3)]. Earlier works have shown that the fieldlike contribution of the spin-orbit torques leads to a distortion of the skyrmion profile but is incapable of moving the skyrmion [10]. Thus, we restrict the following discussion to the case $\beta_{f}=0$, where the force acting on a Néel-type $Q=1$ skyrmion is given by $\boldsymbol{F}^{\text {SOT }} \sim \hat{z} \times \hat{\boldsymbol{P}}$ [17]. If we furthermore assume that $\boldsymbol{P}$ is perpendicular to the injected current, as done, for example, in Ref. [10], the skyrmion Hall angle $\Theta$ follows from our extended Thiele equation [Eq. (6)] at finite temperatures as

$$
\tan \Theta=\frac{\left\langle v_{\perp}\right\rangle}{\left\langle v_{\|}\right\rangle}=\frac{G}{\alpha \mathfrak{D}+\eta T},
$$

where $v_{\perp}$ and $v_{\|}$are the velocity components perpendicular and parallel to the inject current, respectively. The average in the above expression is needed because, at finite temperatures, current-driven skyrmions also undergo Brownian motion, which drops out upon thermal averaging.

In Fig. 4, we compare the simulation results for $\Theta$ with the theoretical prediction (dashed lines) based on Eq. (8). For the sake of visibility, the skyrmion Hall angle is shifted by $\pi / 2$. Note that we use $\beta_{d}=1.25 \times 10^{10} \mathrm{~s}^{-1}$ and, as above, $\eta=5.05 \times 10^{-17} \mathrm{~kg} /(\mathrm{sK})$. Perfect agreement can be observed for $T=0 \mathrm{~K}$, for which our prediction based on Eq. (8) coincides with the well-established prediction $\tan \Theta=G / \alpha \mathfrak{D}$ [9]. We find that the value of $\Theta$ increases with temperature. For example, for $\alpha=10^{-3}$ and $T=24 \mathrm{~K}$, the shifted skyrmion Hall angle is increased by more than 1 order of magnitude compared to the zerotemperature value. The simulation results are in quantitative 
agreement with our theoretical predictions based on Eq. (8), irrespective of $\alpha$ and $T$.

In a recent experimental study [11], a negligible dependence of the skyrmion Hall angle on temperature was reported. However, note that the skyrmions studied in Ref. [11] have a much larger diameter $(\sim 180 \mathrm{~nm})$ compared to those in our simulations. In the experimental skyrmions, only the narrow domain wall around the uniformly magnetized core contributes to the dissipation tensor, so that $\mathfrak{D}$ is proportional to the radius and considerably enhanced in larger skyrmions compared to the nanoscale skyrmions studied here. If the magnon-induced friction scales weaker with the skyrmion size than the dissipation tensor, the latter temperature-independent term would dominate in Eq. (8). While the large experimental skyrmions are not accessible with our simulations, we still varied the diameter of our skyrmions between 2 and $4.5 \mathrm{~nm}$ via the external field. While we did not find a significant dependence of $\eta$ on the skyrmion size within this accessible range, we found that $\mathfrak{D}$ increases with size.

Efforts to derive analytical expressions for the magnoninduced friction by using a field-theoretical approach were made in previous investigations of domain walls [27], vortices [28], and skyrmions [29,30]. In general, the expressions that were derived describe non-Markovian friction, where the friction at time $t$ depends on the history of the velocity. Instead of the simple $\eta T v$ term in Eq. (6), non-Markovian friction is given by $\int_{0}^{t} d t^{\prime} \eta\left(t-t^{\prime} ; T\right) \boldsymbol{v}\left(t^{\prime}\right)$. The question of whether this non-Markovian friction can be effectively treated as Markovian friction is still being debated, e.g., while the authors of Refs. [26,28] claim that assuming Markovian friction is justified, the authors of Refs. $[27,29,30]$ argue that, at least within their approaches, this is not the case. In Ref. [29], it was suggested that including higher-order terms such as magnon-magnon interactions or the backaction of the skyrmion to the magnon bath would give rise to Markovian friction. Our simulation results can be explained by assuming that the magnon-induced friction only has a Markovian contribution.

By looking at skyrmions and the magnonic heat bath in the general context of solitons and their environment, our findings could possibly also be transferred to the dynamics of other localized magnetic textures, e.g., domain walls or vortices, as well as to other topics such as superfluids [44] or Bose-Einstein condensates [45] where similar effects have been investigated.

To summarize, we have studied the Brownian motion and current-driven dynamics of skyrmionic spin structures in the limit of elevated temperatures and low damping. Our results show strong deviations from existing theory based on the Thiele equation. Thus, we introduce an effective equation of motion describing skyrmionic motion at finite temperatures, taking into account the coupling to the magnonic heat bath via an additional friction term that dominates at finite temperatures and low Gilbert damping values. We demonstrate that this magnon-induced friction is linear in temperature and depends on the topological charge of the skyrmionic spin structure.

Having established the validity of our finite-temperature generalization of the Thiele equation, we believe it provides a simple framework to predict the finite-temperature dynamics of skyrmions driven by other means, e.g., spin-transfer torque, magnetic field gradients, or temperature gradients [46]. Our results further indicate a strong temperature dependence of skyrmion dynamics under conditions that are usually found in experiments on the current-driven and Brownian motion of skyrmions. The strength of the magnon-induced friction could be obtained from experiments conducted in a manner similar to the approach presented here, i.e., by measuring the temperature dependence of the skyrmion Hall angle or the diffusion coefficient in low-pinning materials.

The financial support of the Deutsche Forschungsgemeinschaft via Project No. 403502522 and the SFB 1432 and the National Research, Development and Innovation Office of Hungary (Project No. K131938) is gratefully acknowledged.

*Corresponding author. markus.weissenhofer@uni-konstanz.de

[1] A. Bogdanov and A. Hubert, J. Magn. Magn. Mater. 138, 255 (1994).

[2] S. Mühlbauer, B. Binz, F. Jonietz, C. Pfleiderer, A. Rosch, A. Neubauer, R. Georgii, and P. Böni, Science 323, 915 (2009).

[3] N. Nagaosa and Y. Tokura, Nat. Nanotechnol. 8, 899 (2013).

[4] A. Fert, V. Cros, and J. Sampaio, Nat. Nanotechnol. 8, 152 (2013).

[5] D. Pinna, F. Abreu Araujo, J.-V. Kim, V. Cros, D. Querlioz, P. Bessiere, J. Droulez, and J. Grollier, Phys. Rev. Applied 9, 064018 (2018).

[6] Y. Jibiki, M. Goto, E. Tamura, J. Cho, S. Miki, R. Ishikawa, H. Nomura, T. Srivastava, W. Lim, S. Auffret, C. Baraduc, H. Bea, and Y. Suzuki, Appl. Phys. Lett. 117, 082402 (2020).

[7] X. Z. Yu, Y. Onose, N. Kanazawa, J. H. Park, J. H. Han, Y. Matsui, N. Nagaosa, and Y. Tokura, Nature (London) 465, 901 (2010).

[8] N. Romming, C. Hanneken, M. Menzel, J. E. Bickel, B. Wolter, K. von Bergmann, A. Kubetzka, and R. Wiesendanger, Science 341, 636 (2013).

[9] W. Jiang, X. Zhang, G. Yu, W. Zhang, X. Wang, M. Jungfleisch, J. Pearson, X. Cheng, O. Heinonen, K. L. Wang, Y. Zhou, A. Hoffmann, and S. G. E. te Velthuis, Nat. Phys. 13, 162 (2017).

[10] K. Litzius, I. Lemesh, B. Krüger, P. Bassirian, L. Caretta, K. Richter, F. Büttner, K. Sato, O. A. Tretiakov, J. Förster, R. M. Reeve, M. Weigand, I. Bykova, H. Stoll, G. Schütz, G. S. D. Beach, and M. Kläui, Nat. Phys. 13, 170 (2017). 
[11] K. Litzius, J. Leliaert, P. Bassirian, D. Rodrigues, S. Kromin, I. Lemesh, J. Zazvorka, K.-J. Lee, J. Mulkers, N. Kerber, D. Heinze, N. Keil, R. M. Reeve, M. Weigand, B. Van Waeyenberge, G. Schütz, K. Everschor-Sitte, G. S. D. Beach, and M. Kläui, National electronics review 3, 30 (2020).

[12] J. Zázvorka, F. Jakobs, D. Heinze, N. Keil, S. Kromin, S. Jaiswal, K. Litzius, G. Jakob, P. Virnau, D. Pinna, K. Everschor-Sitte, L. Rózsa, A. Donges, U. Nowak, and M. Kläui, Nat. Nanotechnol. 14, 658 (2019).

[13] T. Nozaki, Y. Jibiki, M. Goto, E. Tamura, T. Nozaki, H. Kubota, A. Fukushima, S. Yuasa, and Y. Suzuki, Appl. Phys. Lett. 114, 012402 (2019).

[14] L. Zhao, Z. Wang, X. Zhang, X. Liang, J. Xia, K. Wu, H.-A. Zhou, Y. Dong, G. Yu, K. L. Wang, X. Liu, Y. Zhou, and W. Jiang, Phys. Rev. Lett. 125, 027206 (2020).

[15] A. A. Thiele, Phys. Rev. Lett. 30, 230 (1973).

[16] J. Iwasaki, M. Mochizuki, and N. Nagaosa, Nat. Commun. 4, 1463 EP (2013).

[17] J. Sampaio, V. Cros, S. Rohart, A. Thiaville, and A. Fert, Nat. Nanotechnol. 8, 839 (2013).

[18] C. Schütte, J. Iwasaki, A. Rosch, and N. Nagaosa, Phys. Rev. B 90, 174434 (2014).

[19] R. E. Troncoso and Á. S. Núñez, Ann. Phys. (Amsterdam) 351, 850 (2014).

[20] N. Kerber, M. Weißenhofer, K. Raab, K. Litzius, J. Zázvorka, U. Nowak, and M. Kläui, Phys. Rev. Applied 15, 044029 (2021).

[21] J. Miltat, S. Rohart, and A. Thiaville, Phys. Rev. B 97, 214426 (2018).

[22] M. Weißenhofer and U. Nowak, New J. Phys. 22, 103059 (2020).

[23] A. H. Castro Neto and A. O. Caldeira, Phys. Rev. E 48, 4037 (1993).

[24] J. Iwasaki, A. J. Beekman, and N. Nagaosa, Phys. Rev. B 89, 064412 (2014).

[25] C. Schütte and M. Garst, Phys. Rev. B 90, 094423 (2014).

[26] P. C. E. Stamp, Phys. Rev. Lett. 66, 2802 (1991).

[27] S. K. Kim, O. Tchernyshyov, V. Galitski, and Y. Tserkovnyak, Phys. Rev. B 97, 174433 (2018).

[28] L. Thompson, arXiv:cond-mat/0510414.
[29] C. Psaroudaki, P. Aseev, and D. Loss, Phys. Rev. B 100, 134404 (2019).

[30] C. Psaroudaki, S. Hoffman, J. Klinovaja, and D. Loss, Phys. Rev. X 7, 041045 (2017).

[31] L. Rózsa, A. Deák, E. Simon, R. Yanes, L. Udvardi, L. Szunyogh, and U. Nowak, Phys. Rev. Lett. 117, 157205 (2016).

[32] L. Rózsa, K. Palotás, A. Deák, E. Simon, R. Yanes, L. Udvardi, L. Szunyogh, and U. Nowak, Phys. Rev. B 95, 094423 (2017).

[33] L. Rózsa, M. Weißenhofer, and U. Nowak, J. Phys. Condens. Matter 33, 054001 (2020).

[34] L. D. Landau and E. M. Lifshitz, Phys. Z. Sowjetunion 8, 101 (1935); reprinted in Ukr. J. Phys. 53, Special Issue, 14 (2008), http://archive.ujp.bitp.kiev.ua/files/journals/53/si/ 53SI06p.pdf.

[35] T. L. Gilbert, IEEE Trans. Magn. 40, 3443 (2004).

[36] U. Nowak, Classical spin models, in Handbook of Magnetism and Advanced Magnetic Materials, edited by $\mathrm{H}$. Kronmüller and S. Parkin (John Wiley, New York, 2007), https://doi.org/10.1002/9780470022184.hmm205.

[37] W. F. Brown, Phys. Rev. 130, 1677 (1963).

[38] U. Ritzmann, S. von Malottki, J.-V. Kim, S. Heinze, J. Sinova, and B. Dupé, National electronics review 1, 451 (2018).

[39] J. Slonczewski, J. Magn. Magn. Mater. 159, L1 (1996).

[40] D. Schick, M. Weißenhofer, L. Rózsa, and U. Nowak, Phys. Rev. B 103, 214417 (2021).

[41] J. Barker and G. E. W. Bauer, Phys. Rev. Lett. 117, 217201 (2016).

[42] R. Kubo, Rep. Prog. Phys. 29, 255 (1966).

[43] M. Weißenhofer and U. Nowak, Phys. Rev. B 99, 224430 (2019).

[44] D. K. Efimkin, J. Hofmann, and V. Galitski, Phys. Rev. Lett. 116, 225301 (2016).

[45] P. O. Fedichev, A. E. Muryshev, and G. V. Shlyapnikov, Phys. Rev. A 60, 3220 (1999).

[46] Z. Wang, M. Guo, H.-A. Zhou, L. Zhao, T. Xu, R. Tomasello, H. Bai, Y. Dong, S.-G. Je, W. Chao, H.-S. Han, S. Lee, K.-S. Lee, Y. Yao, W. Han, C. Song, H. Wu, M. Carpentieri, G. Finocchio, M.-Y. Im, S.-Z. Lin, and W. Jiang, National electronics review 3, 672 (2020). 